\title{
Analysis of Factors Causing Motivational Changes of College Students in English Learning
}

\author{
Wei Wang \\ Canvard College, Beijing Technology and Business University, P. R. China
}

Keywords: Motivational Change, Demotivation, Motivational Intensity, Types of Motivation

\begin{abstract}
This paper analyzes the factors influencing the changes in the motivation of college English learners. Six non-English major students voluntarily participated in one-to-one semi-structured interviews. All the salient topics mentioned by the interviewees concerning changes in learning motivation and the causes were marked and common themes were established. The results of the qualitative study confirm the existence of motivational changes that were suggested in the author's preview research, and reveals five causes for the motivational changes: the teacher, (unfavorable) humanistic atmosphere, textbooks, exams, interference of other things. Furthermore, the author studied the relationship between different types of motivation and motivational intensity, with the purpose of solving the problem of motivational intensity decline. The analysis of data indicates that intrinsic interest motivation, information medium motivation, personal development motivation, and social responsibility motivation are positively correlated with motivational intensity, while immediate achievement motivation and short-term planning motivation are negatively correlated with intensity.
\end{abstract}

\section{Introduction}

The current study is based on the author's previous research [1] on motivational changes of Chinese non-English major students in their first two years of college English learning. The research finds are that motivational changes exist in the first two years of college English learning. Generally, most types of motivation (i.e. intrinsic interest motivation, information medium motivation, personal development motivation, and social responsibility motivation) decrease significantly except for the slight increase of immediate achievement motivation, and the decline of motivational intensity is also obvious. Therefore, it is necessary to probe further to find out the causes for the motivational changes as well as some solutions to stop the drop of motivational intensity.

\section{Research Design}

Research Questions. The current study focused on the reasons why there are changes in learning motivations for Chinese non-English major students in their first two years of college English learning. The research questions are: What are the causes for the motivational changes? What are the relationships between different types of motivation and motivational intensity?

Subjects. Six students from Level A, B and C voluntarily participated in the interview study. The purpose of selecting volunteers was to ensure the cooperation of participants [2]. 
Table 1. Demographic Information of the six subjects

\begin{tabular}{|l|l|l|l|l|l|}
\hline No. & Name & Gender & Grade & $\begin{array}{l}\text { CET 4 } \\
(\mathbf{Y} / \mathbf{N})\end{array}$ & $\begin{array}{l}\text { CET 6 } \\
(\mathbf{Y} / \mathbf{N})\end{array}$ \\
\hline 1 & A1 & M & sophomore & Y & Y \\
\hline 2 & A2 & F & sophomore & Y & Y \\
\hline 3 & B1 & M & sophomore & Y & N \\
\hline 4 & B2 & F & sophomore & Y & Y \\
\hline 5 & C1 & M & sophomore & N & N \\
\hline 6 & C2 & F & sophomore & N & N \\
\hline
\end{tabular}

Notes: A---high proficiency, B---middle proficiency, C---low proficiency $\mathrm{F}=$ female $\quad \mathrm{M}=$ male $\quad \mathrm{Y}=$ yes $\quad \mathrm{N}=$ no

Instrument. Semi-structured interview was adopted in the current research. On the one hand, the qualitative study is expected to further confirm the change of motivation in types and intensity, especially to identify demotivation, which were discovered in the previous research [1]; on the other hand, the possible causes for the changes are to be revealed.

"Pearson's product-moment correlation" of SPSS was used in probing the relationship between different types of motivation and motivational intensity.

Data collection. All interviews were carried out by the author in person. According to the time offered by each participant in appointment, the one-to-one interview was conducted in a relaxing atmosphere. With the permission of each interviewee, the whole process was recorded and the recording was switched into transcript later.

Data for "Pearson's product-moment correlation” were obtained from the previous research [1].

\section{Results and Discussion}

Most learning experiences of the six students are the same. They all have reading \& writing class, speaking class as well as in-class online learning class (mainly for listening), use the same textbooks, share equal in-class learning hours each week, take the same tests, etc. However, there is an exception in two aspects: when to participate in CET 4 for the first time and the teachers of the speaking class. All Level A and part of Level B are allowed to take CET 4 earlier than the rest --- at the end of the third semester. Both of the two students (B1 and B2) in the current study took CET 4 at the earlier time. With regard to teachers of the speaking class, students of Level A were taught by foreign teachers, while Level B and C were in the class conducted by Chinese teachers.

Identification of motivational changes. On the question whether there were changes on the reasons why they study English, almost all of the interviewees claimed the influence of exams with the exception of B1 who was under little stress of exams. What's more, it is interesting to find that some of them told that there was no obviously definite reason why to study English in college. They were just accustomed to the way of learning as they did in high school, busy all day along, and this way of learning lasted nearly the first semester. It is evident that motivational types (reasons for learning English) do not maintain the same in the two years. Generally, the motivation of studying for exams becomes evident in the last two semesters. This result is in agreement with the general findings of the quantitative research [1], which gives further support to the identification of changes in motivational types. In detail, the appearance of the motivation studying for cultural reasons and future considerations on Level $\mathrm{A}$ is in accordance with the result in quantitative study that there are significant increases in intrinsic interest motivation (F2), information medium motivation (F3), and personal development motivation (F5) in the second year. What's more, the increase of immediate achievement motivation in the second year is also reflected in interviews with the participants, in particular with B2 and C2. As to the change of motivational intensity, four of the six interviewees clearly stated that the effort they put into English study varied within the two years. 
Between the rest two students, one is B1 who believed that he never put much effort in English study neither at the very beginning of entering university nor in the semester in which he had CET 4/6 to take, and the other is C1 who could not tell whether there was any change on the extent of effort he put into English learning. From their statements, changes on motivational intensity are obvious, and demotivation does exist on some students like A1 and C2 within the two years. However, there are also students whose motivation increases (A2) or fluctuates (B2) in the two years. Due to the small number of the interviewees, it is unreasonable to conclude that their changes on motivation can represent the whole situation, but the changes of motivation can be confirmed from the results of interviews. Considering the large number of questionnaire investigation as well as the feedbacks of most teachers, the result showing demotivation would be more convincing and representative. However, this still needs further probe.

Causes for motivational changes. In interviews, there are five most frequently mentioned categories of factors which can result in motivational changes: the teacher, (unfavorable) humanistic atmosphere, textbooks, exams, interference of other things.

Table 2 The identified main factors causing motivational changes

\begin{tabular}{|ll|l|l|}
\hline \multicolumn{1}{|c|}{ Factors } & Occurrences & Frequency of Occurrences \\
\hline 1. & The teacher & 9 & $36 \%$ \\
\hline 2. & (unfavorable) humanistic atmosphere & 6 & $24 \%$ \\
\hline 3. & Textbooks & 5 & $20 \%$ \\
\hline 4. & Exams & 3 & $12 \%$ \\
\hline 5. & Interference of other things & 2 & $8 \%$ \\
\hline
\end{tabular}

The table 2 lists the negative/positive influence mentioned by at least two students as the main sources of their demotivation or factors that could negatively/positively affect their English study. The five categories account for a total of 25 corresponding occurrences in the transcripts. The largest category (with $36 \%$ of the total frequency of occurrences) directly concerned the "teacher", including teaching method, competence, personality, which is consistent with the results reported by other researchers $[3,4,5]$. From the sample extracts in this category, we can find both negative and positive influence from teachers. That is, negative influence can lead to demotivation in English learning and positive influence can function oppositely. Another significant proportion (24\%) of the demotives were accounted for by "(unfavorable) humanistic atmosphere" which is complementary to the "inadequate school facilities" by Dörnyei [5] who claimed "objective school conditions can constitute an 'affective filter' that is powerful enough to block even the best intentions on the students' or teachers' part” [6]. And this is also true that the inadequacy of abstract cultural and humanistic atmosphere can also pose a similar "affective filter" to discourage learners. The category of textbook (20\%) shows that the textbooks used currently cannot meet the demand of students and this can result in demotivation, or at least not be helpful for promoting motivation, though there are a lot of improvements in the current textbook compared with the previous ones. The last two categories are unique to the investigation of Chinese students on demotivation. In other words, they have never been mentioned by researchers in other countries. Chinese students have to undergo many English tests which make them suffer a lot. Furthermore, the English testing system in China is unsatisfactory, with many potential problems. Therefore, it is easy to understand why exams can exert negative influence on English learning. The last category is also closely related to the development of Chinese society. Nowadays, in China, "interpersonal relation" is of great importance in life and work because a good network of relations can make almost everything easy. And this is also realized by college students as they are studying in college which is the preparatory stage for stepping into society. Therefore, it is natural to understand why they would invest less and less effort in study. 
Table 3 Correlation between types of motivation and motivational intensity

\begin{tabular}{|l|l|l|l|l|l|l|l|l|}
\hline \multicolumn{2}{|c}{} & F1 & F2 & F3 & F4 & F5 & F6 & F7 \\
\hline \multirow{2}{*}{$\begin{array}{l}\text { INTENSIT } \\
\text { Y }\end{array}$} & $\begin{array}{l}\text { Pearson } \\
\text { Correlation }\end{array}$ & -.011 & $.538(* *)$ & $.408(* *)$ & $-.291\left(^{* *}\right)$ & $.359\left(^{* *}\right)$ & $.216\left(^{* *}\right)$ & $-.117\left(^{* *}\right)$ \\
\cline { 2 - 9 } & Sig. (2-tailed) & .746 & .000 & .000 & .000 & .000 & .000 & .000 \\
\cline { 2 - 9 } & $\mathrm{N}$ & 856 & 856 & 856 & 856 & 856 & 856 & 856 \\
\hline
\end{tabular}

F1 = Learning situation, F2 = Intrinsic interest, F3 = Information medium,

F4 = Immediate achievement, F5 = Personal development, F6 = Social responsibility

F7 = Short-term planning

As shown in table 3, motivational intensity positively correlates with intrinsic interest motivation (F2), information medium motivation (F3), personal development motivation(F5), and social responsibility motivation(F6) in a significant way, and the correlationcoefficientsare0.538,0.408,0.359,and 0.216 respectively at the 0.01level (2-tails). The results are consistent with Gao et al' $\mathrm{s}$ [7] findings which also suggest the positive relation between intensity and motivational types of interest, information medium, personal development, social responsibility. In addition, the findings in the current research once more prove the supreme importance of initiating interest for foreign language learning which has been claimed numerously in previous researches, and also indicate the impact of some instrumental motivation like information medium and personal development on the extent of effort students are willing to put in English study. The influence from the collaboration of cultural and instrumental motivation on English learning, in some degree, testifies Gardner' $s$ [8] claim that both integrative and instrumental motivation can facilitate successful L2 learning. In contexts where learners have few or no opportunities to interact with members of the target language (such as China), instrumental motivation would be more powerful, and this has been proved in previous researches [9, 10]. Generally, students can be motivated to spend more efforts on English learning by effectively arousing their interest towards the language and its culture, making them often personally experience the utilitarian function of English in their real life and constantly deepen their understanding of the potential usefulness of the language for their personal development, as well as frequently emphasizing their duty to fulfill the social responsibility of making the world know more about China and living up to their parents expectations.

Apart from these positive correlations, the current study also suggests the significant negative correlation between motivational intensity and immediate achievement motivation(F4), short-term planning motivation(F7).The corresponding correlation coefficients are -0.291 and -0.117 at the 0.01 level (2-tails). The results indicate that instrumental motivation should be employed in a selective way to maximize its effects on strengthening motivational intensity. In addition, the unique feature of language learning could also be the possible cause for the positive relation between intensity and information medium motivation, personal development motivation, as well as the negative one between intensity and immediate achievement motivation, short-term planning motivation. Language learning is along, laborious and constant process, and it is very important to establish regular and long-term study program. Motivations producing immediate results may not be good for sustaining efforts in a long run, and once the short-term goals are realized, the effect from this kind of motivation would cease. However, the connection between the feature of languagelearningandinstrumentalmotivationneedfurtherstudytoprobe.

Unexpectedly, there is no significant correlation existing between intensity and learning situation motivation. Namely, teachers, text books, English classes, etc. do not cause much influence on the amount of effort students put in their English study. In other words, how much effort students will spend does not depend much on the quality of classes or other elements of learning situation. This result is quite contradictory to the findings of previous researches which consistently highlighted the positive or negative influence from teachers (or other related factors like teaching methods) on 
enhancing motivation or demotivation $[3,4,5,11]$. However, this result could justify the second explanation for the stability of learning situation motivation for most students in the two years, which suggests that students are independent of the influence from the components of learning situation. It is very likely that the loose connection between the two may result from students becoming more and more independent in college, and this needs to be further confirmed. From the correlation study between different types of motivation and motivational intensity, we could also find out the connection between demotivation and the change of motivational types. Since there are positive correlations between intensity and intrinsic interest (F2), information medium motivation (F3), personal development motivation (F5), and social responsibility motivation (F6), the decline in these motivations would result in the drop in intensity. Therefore, in order to effectively inhibit the occurrence of demotivation in English learning, measures should be taken to prevent the decrease of positively related motivations(F2, F3, F5, andF6). Meanwhile, the negatively related motivations should also be controlled in a proper way.

\section{Conclusion}

The current study is complementary to the author's previous research on motivational changes. The research findings reveals that factors from the five aspects could result in motivational changes, in particular demotivation: teacher, (unfavorable) humanistic atmosphere, textbooks, exams and interference of other things. What's more, intrinsic interest motivation, information medium motivation, personal development motivation, and social responsibility motivation are positively correlated with motivational intensity, while immediate achievement motivation and short-term planning motivation are negatively correlated with intensity. Therefore, as long as these factors and the positive/negative relations are well controlled, it is likely that the college students' English learning motivation will be effectively promoted.

\section{References}

[1] Wang Wei, Study on motivational changes of non-English majors, International English Education Research. 5 (2016) 49-51.

[2] Wen, Qiufang, The Changing pattern and characteristics of English learners' motivation, concept and strategies, Foreign Language Teaching and Research. 2 (2001) 105-110.

[3] Oxford, R., Language learning motivation: expanding the theoretical framework, Modern Language Journal. 78(1994)12-28.

[4] Ushioda, E., Effective motivational thinking: A cognitive theoretical approach to the study of language learning motivation. In Soler, E.A.andEspurz, V.C.(Eds.) , Current Issues in English Language Methodology, Unversitat Jaum I, CastellódelaPlana, Spain, 1998, pp.77-89.

[5] Dörnyei, Z.Demotivation in foreign language learning. Paper presented at the TESOL'98Congress,Seattle,WA,March. (1998)

[6] Dörnyei, Z., Teaching and Researching Motivation, Beijing: Foreign Language Teaching and Research Press, Beijing, 2005.

[7] Gao, Yihong. et. al., Social Psychology of Chinese College Students' English Learning--Learning Motivation \&Self-identity, Foreign Language Teaching and Research Press, Beijing, 2004.

[8] Gardner, R.C., Social Psychology and Second Language Learning: The Role of Attitudes and Motivation, EdwardArnold Publishers, London, 1985.

[9] Gardner, R. C., \& Lambert, W. E., Attitudes and Motivation in Second Language Learning, Newbury House, Rowley, 1972. 
[10] Lukmani, Y. M., Motivation to learn and learning proficiency. Language Learning. 22(1972)261-73.

[11] Chambers, N. G., Talking the 'de' out of demotivation. Language Learning Journal. 7(1993)13-16. 